\title{
Aortic Isthmus Aneurysm: A Rare Presentation
}

\author{
S Chand, R Chauhan \\ Department of General Medicine, Dr. Radha Krishnan Govt. Medical College and Hospital, Hamirpur, Himachal Pradesh, India.
}

\author{
Corresponding Author: \\ Dr Subhash Chand \\ Email: subhashchand964@yahoo.com \\ This is an Open Access article distributed \\ under the terms of the Creative Commons \\ Attribution License (creativecommons.org) \\ licenses/by/3.0). \\ Published

\begin{abstract}
Background: An aneurysm is enlargement of artery more than 1.5 times its normal diameter. Thoracic aortic aneurysms involving isthmus are very rare. Most of these aneurysms are asymptomatic and slow growing. Aortic dissection and rupture are known complications causing sudden death. Case Report: In this short communication, we present a case of 45 year old female who presented with repeated episodes of palpitations and on investigations thoracic aortic aneurysm was found. Conclusion: Homogenous opacity in the region of aortic arch can be due to aortic aneurysm. CT angiography is done to confirm the diagnosis. Endovascular repair of aneurysm is the treatment of choice with good results.
\end{abstract}

Keywords: Arteries, Aortic Aneurysm, Female, Sudden Death.
A 45-year-old female, known case of hypertension presented with repeated episodes of palpitation. Chest X-ray of the patient was showing a homogenous opacity in the region of aortic arch [Fig.1] and on CT chest an aortic aneurysm was found. Subsequently upon CT angiography of aorta at higher centre, aortic isthmus/proximal descending thoracic aortic aneurysm measuring $5.7 \mathrm{~cm}$ in maximum calibre for a length of $4.4 \mathrm{~cm}$ was seen [Fig.2]. Thoracic endovascular aortic repair (TEVAR) was done successfully at higher centre and patient is doing well.

An aortic aneurysm is defined as enlargement of aorta to greater than 1.5 times the normal size [1]. The normal diameter of the ascending aorta is $<2.1 \mathrm{~cm} / \mathrm{m}^{2}$. and descending aorta is $<1.6 \mathrm{~cm} / \mathrm{m}^{2}$. Dilation of the aorta can be localised or diffuse. Diffuse dilation of the artery is called as arteriomegaly. If the dilation of the artery is less than $50 \%$ it is called as ectasia. Thoracic aortic aneuryms have an incidence of 5-10 per 100,000 person-years [2]. Thoracic aortic aneurysms are classified into aortic root aneurysms, ascending aortic aneurysms which are most common about $60 \%$ followed by aneurysms of descending aorta $35 \%$ and aneurysms of aortic arch which are less than $10 \%$.

Aortic aneurysm can be due to connective tissue disorders like Marfan's syndrome and Ehler Denlos syndrome, bicuspid aortic valve disease, atherosclerosis and infections. Thoracic

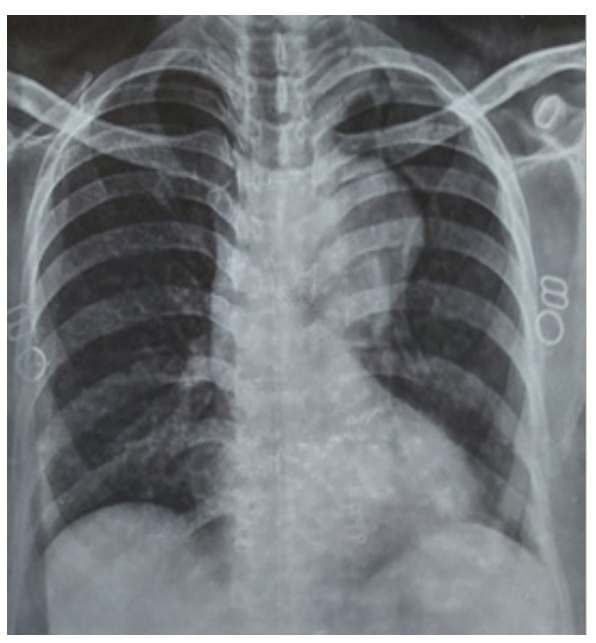

Fig.1: Chest $x$-ray showing a homogenous opacity in the region of aortic arch. 
aortic aneurysms usually occur in the age group of 60-70 years. Hypertension and smoking are the most common risk factors. Approximate $10 \%$ of patients can have family history of aortic aneurysm. Individuals with history of aneurysm in other parts of body have higher incidence of developing thoracic aortic aneurysm. Thoracic aortic aneurysms often grow slowly usually without symptoms, making them difficult to detect. Common symptoms are pain in the chest and back, hoarseness of voice, cough or shortness of breath. Growth of aneurysm cannot be predicted.

Early diagnosis of thoracic aneurysm is critical to managing the condition. The larger the thoracic aortic aneurysm or the faster it grows the more likely it is to rupture. The risk of rupture is higher when size of the blood vessel grows twice the normal. Diagnosis of thoracic aortic aneurysm is difficult because most of the patients are asymptomatic and the condition goes undiagnosed until it ruptures. Suspicion of aortic aneurysm is usually made during routine investigation like chest X-ray, CT scan chest or echocardiography. $\mathrm{CT}$ angiography or MRI angiography are done to confirm the diagnosis.

Surgery is the definitive treatment of an aortic aneurysm. Medical therapy is typically reserved for smaller aneurysms or for elderly frail patients. Medical treatment consists of control of blood pressure. This per se will not treat the aneurysm but will decrease the rate of expansion of aneurysm. Ultrasound studies are done on regular basis to follow the size of the aneurysm. Surgery either open or endovascular is the definitive treatment for aortic aneurysm. Decision for repairing an aortic aneurysm is based on the balance between the risk of rupture and risk of treatment itself. Endovascular treatment of aortic aneurysms is minimally invasive and is alternative to open surgical repair [3]. It involves placement of an endovascular stent. Endovascular aneurysm repair has shorter hospital stay and low risk of death.

In this case we figured out that aortic

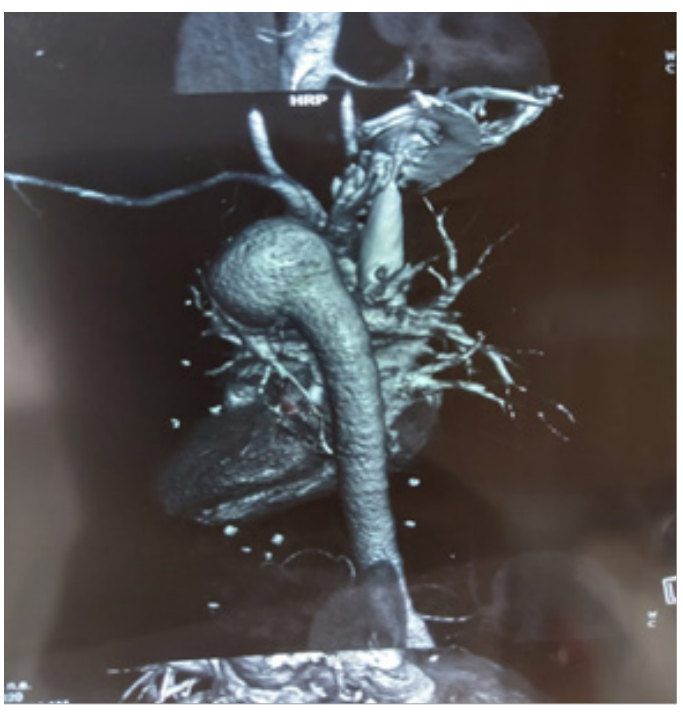

Fig.2: CT angiography showing aortic isthmus/proximal descending thoracic aortic aneurysm.

aneurysm does not produce specific symptoms. Homogenous opacity in the region of aortic arch can be due to aortic aneurysm. CT angiography is done to confirm the diagnosis. Endovascular repair of aneurysm is the treatment of choice with good results.

Contributors: SC: Patient management and preparation of draft manuscript; $\mathrm{RC}$ : patient management and manuscript revision. SC acted as study guarantor. Both authors approved the final version of the manuscript and are responsible for all aspects of the study.

Funding: None; Competing interests: None stated.

\section{References}

1. Johnston KW, Rutherford RB, Tilson MD, Shah DM, Hollier L, Stanley JC. Suggested standards for reporting on arterial aneurysms, Subcommittee on Reporting Standards for arterial Aneurysms, Ad Hoc Committee on Reporting Standards, Society for Vascular Surgery and North American Chapter, International Society for Cardiovascular Surgury. Journal of Vascular Surgery. 1991;13(3):452-458.

2. Kuzmik GA, Sang AX, Elefteriades JA. Natural history of thoracic aortic aneurysms. J Vasc Surg. 2012:56:565.

3. Thomas Dustin M, Hulten Edward A, Ellis Shane T, Anderson David MF, Anderson N, McRae Fiora, et al. Open versus endovascular repair of abdominal aortic aneurysm in the elective and emergent setting in a pooled population of 37,781 patients: A Systemic Review and Meta-analysis". ISRN Cardiology. 2014: Article ID:149243. 